Retraction

\title{
Retraction: Yang, C., et al. miR-126 Functions as a Tumor Suppressor in Osteosarcoma by Targeting Sox2. Int. J. Mol. Sci. 2014, 15, 423-437, doi:10.3390/ijms15010423
}

\author{
Chenglin Yang ${ }^{1}$, Chunying Hou ${ }^{1}$, Hepeng Zhang ${ }^{1}$, Dewei Wang ${ }^{1}$, Yan Ma ${ }^{1}$, Yunqi Zhang ${ }^{1}$, \\ Xiaoyan $\mathrm{Xu}^{1}{ }^{1}$, Zhenggang $\mathrm{Bi}^{1}{ }^{1}$, Shuo Geng ${ }^{1}$ and International Journal of Molecular Sciences \\ Editorial Office ${ }^{2}$ \\ 1 Department of Orthopedic Surgery, the First Affiliated Hospital of Harbin Medical University, \\ Harbin 150001, Heilongjiang, China; yangchenglindoctor@gmail.com (C.Y.); chunying_hou@126.com (C.H.); \\ zhanghepengdoctor@gmail.com (H.Z.); wdw19761224@gmail.com (D.W.); mayandoctor@gmail.com (Y.M.); \\ zhangyunqidoctor@gmail.com (Y.Z.); xxydoctor@gmail.com (X.X.); bizhenggang@163.com (Z.B.); \\ shuogeng.hmu@gmail.com (S.G.) \\ 2 MDPI AG, St. Alban-Anlage 66, 4052 Basel, Switzerland; ijms@mdpi.com
}

Received: 18 October 2018; Accepted: 18 October 2018; Published: 10 November 2018

Experimental results from [1] were found to be unreliable. In particular, the authors have attempted to replicate the real-time PCR and Western blot experiments but were not able to obtain the same results. All of the authors have agreed to the retraction of the paper [1], which will thus be marked online as retracted. The International Journal of Molecular Sciences is a member of the Committee on Publication Ethics (COPE) and takes very seriously the responsibility to enforce strict ethical policies and standards.

\section{Reference}

1. Yang, C.; Hou, C.; Zhang, H.; Wang, D.; Ma, Y.; Zhang, Y.; Xu, X.; Bi, Z.; Geng, S. miR-126 Functions as a Tumor Suppressor in Osteosarcoma by Targeting Sox2. Int. J. Mol. Sci. 2014, 15, 423-437. [CrossRef] [PubMed]

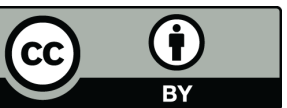

(C) 2018 by the authors. Licensee MDPI, Basel, Switzerland. This article is an open access article distributed under the terms and conditions of the Creative Commons Attribution (CC BY) license (http:/ / creativecommons.org/licenses/by/4.0/). 